\title{
Usefulness Of A Commercial LAMP Assay For Detection Of Malaria Infection, Including Plasmodium Knowlesi Cases, In Returning Travelers In Spain
}

\section{Alexandra Martín Ramírez}

Carlos III Health Institute: Instituto de Salud Carlos III

Marta Lanza

Carlos III Health Institute: Instituto de Salud Carlos III

\section{Shamilah Hisam}

Institute for Medical Research. National Institute of Health.

Ana Perez-Ayala

Hospital Universitario 12 de Octubre

José M. Rubio ( $\square$ jmrubio@isciii.es )

Carlos III Health Institute: Instituto de Salud Carlos III https://orcid.org/0000-0002-1903-6711

\section{Research Article}

Keywords: Malaria, Plasmodium, molecular diagnosis, Plasmodium knowlesi, LAMP, illumigene® Malaria, Alethia ${ }^{\circledR}$ Malaria, PCR.

Posted Date: February 28th, 2022

DOI: https://doi.org/10.21203/rs.3.rs-1326927/v1

License: (c) (i) This work is licensed under a Creative Commons Attribution 4.0 International License.

Read Full License 


\section{Abstract}

Objective: Main malaria diagnosis is based on microscopic examination combined with rapid diagnostic tests. Both methods have low sensitivity and specificity. Loop-mediated isothermal amplification techniques have shown a sensitivity similar to PCR but with lower times of performance.

This study aimed to assess a commercial LAMP for the diagnosis of malaria (Alethia ${ }^{\circledR}$ Malaria) against the Nested-Multiplex-Malaria PCR, including the analytical sensitivity and the operational characteristics.

Results: One hundred five samples out of 114 rendered valid results, obtaining 85 positive samples and 18 negative samples with an agreement of $98 \%$ compared to the reference method with a sensitivity, specificity and kappa coefficient of $98.84 \%, 94.74 \%$ and 0.94 respectively, with only two discrepant samples. The turnaround time was estimated in 1 hour and 30 minutes, with a cost of $32.67 €$ per determination. The results showed several advantages of the Alethia ${ }^{\circledR}$ Malaria, as it was easy to perform, minimal training requirement and 40 minutes run. Moreover, it includes an internal control to avoid false negatives. However, it also showed some limitations such as the need for a specific amplification and detection device, the detection of only Plasmodium spp. and a very high price.

\section{Introduction}

According to the last World Malaria Report, in 2020 an estimated 241 million cases of malaria occurred worldwide [1]. On the way towards elimination, one of the major issues to be addressed is the development of highly sensitive, reliable and easy-to-perform methods for the point-of-care diagnosis of malaria $[1,2]$.

In non-endemic countries, the majority of cases are imported [3] and locally acquired infections are rare events [4]. In Spain, malaria was declared officially eliminated in 1964 [5]. Since then, all reported cases have been imported, except for a few cases of locally acquired malaria [6].

Main malaria diagnosis is based on microscopic examination of Giemsa-stained thick and thin blood smears, often combined with rapid diagnostic tests (RDTs) [7]. Both methods have low sensitivity, unable to detect cases with low parasitaemia and/or asymptomatic and low specificity, not detecting the species involved or requiring expert microscopists $[2,7]$.

Nucleic acid-based detection of malaria parasites (NAT) is now routine in reference laboratories of several non-malaria endemic countries, mostly based on polymerase chain reaction (PCR) [7]. However, novel techniques based on isothermal methods have been designed [8-10]. LAMP method amplifies DNA with high specificity, efficiency and rapidity under isothermal conditions [11]. The strength of this technique relies on the possible application to low resource settings because it can be performed without specific expensive devices, just with a simple heating block or water bath [12]. There are several LAMP commercial kits developed to detect malaria parasites $[10,13,14]$. Alethia ${ }^{\circledR}$ Malaria LAMP assay (Meridian Bioscience), previously called illumigene ${ }^{\circledR}$ Malaria, is a qualitative in vitro diagnostic LAMP 
test for detection of Plasmodium spp. that has been tested in endemic [13] and in non-endemic malaria countries $[2,14-16]$. However, most of the published studies include none or very few samples of $P$. knowlesi parasites $[16,17]$.

This study aimed to assess the diagnostic performance of the Alethia ${ }^{\circledR}$ Malaria LAMP assay for the five species of Plasmodium that infect humans commonly, including $P$. knowlesi, compared to the NestedMultiplex-Malaria PCR (NM-PCR). This evaluation includes the performance of the tests, the analytical sensitivity and the operational characteristics.

\section{Material And Methods}

\section{Clinical samples and study design}

Samples used belonged to a project approved by the Medical and Health Research Ethics Committee (CEIC) of the Hospital Universitario 12 de Octubre (No. CEtm: 18/021). Plasmodium knowlesi samples were collected in Malaysia under a project approved by the Medical Research Sub-Committee of the Malaysian Ministry of Health (NMRR-13-1064-18189). One hundred fourteen samples, 93 malaria positive (65 P. falciparum, 6 P. vivax, 9 P. ovale, 4 P. malariae and 9 P. knowlesi) and 21 negative samples were tested.

\section{Alethia ${ }^{\circledR}$ Malaria LAMP assay}

Alethia ${ }^{\circledR}$ Malaria LAMP kit includes the material to perform the DNA extraction and the LAMP reaction. The assay was performed according to the manufacturer's instructions using the Illumipro- $10^{\mathrm{TM}}$ (Meridian Bioscience) for the amplification and reading of the results, obtaining a positive, negative or invalid result in 40 minutes. The LAMP reaction consists of two tubes with all the lyophilized components, one for the amplification of Plasmodium spp. and the second as internal reaction control.

\section{Nested-Multiplex-Malaria PCR (NM-PCR)}

DNA purification from $200 \mu$ of whole EDTA blood was performed using the QIAamp DNA mini blood kit (QIAGEN ${ }^{\circ}$, Inc.) according to the manufacturer's instructions.

NM-PCR was performed using gel form tubes (BioMalar Kit, Biotools) according to the manufacturer and original authors' recommendations $[18,19]$. The method involves two multiplex PCR amplifications that target the small subunit rDNA gene of Plasmodium. The first reaction amplifies Plasmodium spp. and an internal amplification control and the second reaction enables the identification of the infecting species by the corresponding size of the amplified fragments in the agarose gel electrophoresis [19].

The parameters used for the amplification were an initial denaturation at $94^{\circ} \mathrm{C}$ for $7 \mathrm{~min}$, followed by 40 cycles at $94^{\circ} \mathrm{C}$ for 20 seconds, $58^{\circ} \mathrm{C}$ for 20 seconds, and $72^{\circ} \mathrm{C}$ for 30 seconds. The last cycle was followed by an extension time at $72^{\circ} \mathrm{C}$ for 10 minutes. Conditions for the second PCR reaction were an 
initial denaturation at $94^{\circ} \mathrm{C}$ for 5 minutes, followed by 35 cycles at $94^{\circ} \mathrm{C}$ for 15 seconds, $53^{\circ} \mathrm{C}$ for 15 seconds, and $72^{\circ} \mathrm{C}$ for 20 seconds, finishing with an extension phase at $72^{\circ} \mathrm{C}$ for 10 minutes.

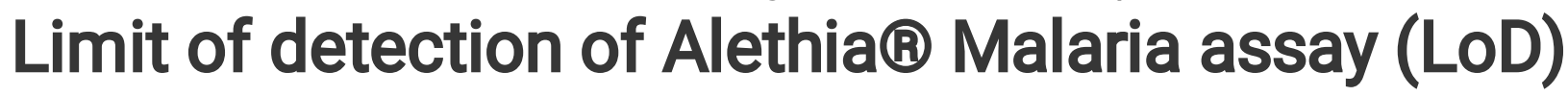

LoD was determined, by duplicate, using a ten-fold serially diluted $P$. falciparum positive blood sample belonging to a patient with a parasitaemia of 7,500 parasites/ $\mu$ determined by microscopy. LoD was considered as the lowest parasite concentration in which both duplicates were positive.

\section{Operational characteristics}

Estimated costs for the Alethia ${ }^{\circledR}$ Malaria assay and the NM-PCR method were referred to as the cost for reagents for one determination without including costs associated with labor or derived from duplicates or controls. Turnaround time was estimated as the time required from the initial moment the sample begins to be processed until obtaining the results, while hands-on work time was the time required for the staff to perform the assay.

\section{Statistics}

Sensitivity (S), specificity (E), positive (PPV) and negative predictive value (NPV) and Kappa coefficient (k) were analyzed with 95\% confidence intervals using EPI Dat (3.1) software package [20].

\section{Results}

One hundred five samples out of 114 rendered valid results while nine samples gave invalid results, when Alethia ${ }^{\circledR}$ Malaria assay was used, obtaining 85 positive samples and 18 negative samples with an agreement of $98 \%$ compared to the reference method (Table 1). Only two samples (1.9\%) provided discrepant results, a P. malariaeinfected sample characterized by the NM-PCR, which was negative by the LAMP method, and a negative sample which rendered a positive result by the LAMP (Table 1).

Table 1

Comparison of the Alethiaß Malaria kit and NM-PCR results.

\begin{tabular}{|c|c|c|c|c|c|c|c|}
\hline \multirow{2}{*}{$\begin{array}{l}\text { Alethia }{ }^{\circledR} \\
\text { Malaria }\end{array}$} & \multicolumn{7}{|l|}{ NM-PCR } \\
\hline & $\begin{array}{l}P \\
\text { falciparum }\end{array}$ & $\begin{array}{l}P \\
\text { ovale }\end{array}$ & $\begin{array}{l}P . \\
\text { vivax }\end{array}$ & $\begin{array}{l}P . \\
\text { malariae }\end{array}$ & $\begin{array}{l}P \\
\text { knowlesi }\end{array}$ & Negative & Total \\
\hline Positive & 59 & 9 & 6 & 2 & 9 & 1 & 86 \\
\hline Negative & 0 & 0 & 0 & 1 & 0 & 18 & 19 \\
\hline Invalide & 6 & 0 & 0 & 1 & 0 & 2 & 9 \\
\hline Total & 65 & 9 & 6 & 4 & 9 & 21 & 114 \\
\hline
\end{tabular}

The values of sensitivity and specificity were $98.8 \%$ and $94.7 \%$ respectively and the Kappa coefficient was 0.94 (Table 2). 
Table 2

Sensitivity, specificity, predictive values and kappa coefficient of Alethia ${ }^{\circledR}$ Malaria assay compared to NMPCR.

\begin{tabular}{|lll|}
\hline & Value (\%) & $\mathbf{9 5 \%}$ Cl \\
\hline Sensitivity & 98.84 & $95.99-100$ \\
\hline Specificity & 94.74 & $82.06-100$ \\
\hline Positive predictive value & 98.84 & $95.99-100$ \\
\hline Negative predictive value & 94.74 & $82.06-100$ \\
\hline Kappa coefficient & 0.94 & $0.85-1$ \\
\hline Cl: confidence interval & & \\
\hline
\end{tabular}

The limit of detection of the Alethia $\Theta$ Malaria assay was 0.075 parasites $/ \mu$ l.

The turnaround time for the Alethia ${ }^{\circledR}$ Malaria assay was estimated in 1 hour 30 minutes $(30$ minutes for DNA purification, 10 minutes for tubes preparation, 40 minutes for amplification and 10 minutes for results reading), while for NM-PCR, it was estimated in 6 hours 15 minutes (1 hour for the management of the samples and DNA purification, 15 minutes for the first PCR setup, with the tubes ready to use, 2 hours to run the first PCR, 15 minutes for the second PCR setup, 2 hours to run the second PCR, 30 minutes for the automated electrophoresis, and 15 minutes for the analysis of results).

The cost for the commercial assay was calculated as $32.67 €$, higher than for the "in-house" assay of $5 €$, although in other countries or institutions, the costs of kits may be lower (Table 3 ).

Table 3

Time and costs estimated for Alethia $B$ Malaria and NM-PCR assays.

\begin{tabular}{|llll|}
\hline Technique & Turnaround time & Hands-on work & Costs per determination \\
\hline Alethia $\circledast$ Malaria assay & 1 hour 30 minutes & 50 minutes & $32.67 €$ \\
\hline NM-PCR & 6 hours 15 minutes & 2 hours & $5.00 €$ \\
\hline
\end{tabular}

\section{Discussion}

There is a growing request for faster and more sensitive diagnostic methods for malaria. Conventional methods, such as microscopy and RDTs, lack sensitivity, while PCR-based methods, although very sensitive [7], require highly trained personnel and expensive equipment. In contrast, LAMP-based methods have proven to have a similar or higher sensitivity than PCR methods but with the need for less training and resources $[16,21]$. Alethia $\AA$ Malaria kit is a qualitative commercial assay able to detect Plasmodium 
spp. based on LAMP technology [22, 23]. Furthermore, the kit includes the components to isolate DNA from whole blood by centrifuge-free methods [13]. In our study, the comparison between LAMP and NMPCR assays, with just two discrepant samples (1.9\%), showed a very good correlation corroborated with the high values of sensitivity, specificity and predictive values, as well as the kappa value (Table 2). Regarding the two discrepant results, it is very difficult to assess the accuracy of the LAMP method because any meaningful evaluation must be involved in comparison with other methods of diagnosis, in this occasion the NM-PCR, which might themselves be wrong. The false-positive result could be due to DNA contamination during sample processing $[24,25]$ or even to be a true-positive [10]. In each extraction set, a free-DNA sample was included and it did not produce amplification by the LAMP method, so possibly the second option was more feasible. The false-negative result in the sample infected with $P$. malariae may be due to a low parasitaemia of the original sample or that the LAMP assay exhibits deficiencies for the detection of this species. In this study, only four $P$. malariaeinfected samples were analyzed, of which only two (50\%) were correctly characterized, meanwhile another was being considered negative and the last gave an invalid result. Expanding the number of samples infected with $P$. malariae would be necessary to find the correct answer.

Similar good results in specificity and sensitivity have been shown in more studies in non-endemic countries $[2,14,15,17]$. In a study performed in France [2], they obtained $100 \%$ of sensitivity and $98.13 \%$ specificity using real-time PCR as the reference method. Studies performed in North America obtained excellent sensitivity compared to microscopy [14] and PCR [16]. Moreover, the Alethia $®$ Malaria kit has also been evaluated in Senegal obtaining high sensitivity (97.2\%) and a good specificity [13]. However, neither of these studies tested any $P$. knowlesi specimens. In our study, the nine $P$. knowlesi-infected samples were detected, without any invalid or false-negative results, confirming the ability of the Alethia ${ }^{\circledR}$ Malaria LAMP to detect the main five Plasmodium human species.

Nine samples out of 114 provided invalid results. Several authors have described a similar situation where it was not possible to obtain a valid result with this LAMP assay $[2,16]$. According to the manufacturer's instructions, some possible reasons for the invalid results may be inhibitory specimens, improper sample preparation, reagent failure, instrument failure, dirty device or improperly seated. In our study, samples with an invalid result were obtained at the beginning of the study but not at the end, suggesting that the technologist's level of training played a factor in the results probably due to improper mixing of the blood samples and buffers.

The Alethia $\circledast$ Malaria kit showed a very good LoD value for $P$. falciparum, 0.075 parasites $/ \mu$, similar to reported for several malaria Nested PCRs $[18,26]$. Other reports obtained different LoD depending, possibly, on the origin of the samples used; 2 parasites/ $\mu \mathrm{l}$ [12] and 0.5 parasites/ $\mu \mathrm{l}$ [15] with conditions similar to ours, or 0.1 parasites $/ \mu$ l using serial dilutions of $P$. falciparum cultures [2].

Several malaria LAMP assays have been described previously [8-11], most of them "homemade" assays with reproducibility problems and with difficult-to-pass quality assessment controls. Conversely, commercial LAMP kits for malaria detection present the reagents in lyophilized form to enhance stability 
under ambient conditions, facilitating the use and decreasing the need for high training. Furthermore, the Alethia $B$ Malaria kit incorporates the components to purify DNA from whole blood and an internal reaction control to discriminate false negatives from inhibition reactions. Unfortunately, contrary to the best feature of LAMP technology, the reaction must be run on a specific device. Despite this, given our results, with excellent sensitivity and specificity and with a reduced time of diagnosis, Alethia ${ }^{\circledR}$ Malaria assay could be used as a good screening diagnosis method for malaria, as other authors have pointed out previously $[12,16,17]$.

\section{Conclusions}

In this study, we demonstrated the utility of the Alethia ${ }^{\circledR}$ Malaria. Our evaluation showed several advantages, as it was easy to perform, minimal training was needed, DNA purification was simple, fast and included in the kit, and the amplification run was completed in 40 minutes. Moreover, it includes an internal control to avoid false negatives. However, it also showed some limitations such as the need for a special amplification and detection device, the detection of only Plasmodium spp. with no information about species or the level of parasitaemia and the higher price compared to non-commercial assays.

\section{Limitations}

Our study presented some limitations, as the retrospective analysis of the samples and the low number of samples evaluated, especially for non-falciparum species. In addition, mixed infections were not included in the assessment and we did not undertake a reproducibility analysis.

\section{List Of Abbreviations}

EDTA: ethylenediaminetetraacetic acid; LAMP: Loop-mediated isothermal amplification; LoD: Limit of Detection; PCR: Polymerase Chain Reaction; NM-PCR: Nested-Multiplex-Malaria PCR; RDT: rapid diagnostic test.

\section{Declarations}

\section{Ethics approval and consent to participate}

Samples used in this study belonged to travelers or immigrants coming from endemic malaria areas that attended different Spanish hospitals with malaria or other tropical diseases suspicion. They were sent to the Spanish Malaria Reference Center within a project for the detection of submicroscopic malaria cases approved by the Medical and Health Research Ethics Committee (CEIC) of the Hospital Universitario 12 de Octubre (No. CEtm: 18/021). The participants of this project were informed of the objectives and expected results and signed informed consent for their participation. Plasmodium knowlesi samples were collected in Malaysia under a project approved by the Medical Research Sub-Committee of the Malaysian Ministry of Health (NMRR-13-1064-18189). 


\section{Consent for publication}

Not applicable

\section{Availability of data and materials}

The databases used and/or analyzed during the current study are available from the corresponding author on reasonable request.

\section{Competing interests}

The authors declare that they have no competing interests.

\section{Funding}

This work was partially supported by the Spanish Strategic Health Action (AESI-ISCIII) Grant Number PI17CIII/00035. The funder had no role in study design, data collection and analysis, decision to publish, or preparation of the manuscript. Alexandra Martín Ramírez is supported by an ISCIII Río Hortega grant.

\section{Authors' contributions}

JMR, AMR, APA, conceptualized the study. JMR, AMR, designed the experiments; JMR provided training and supervision for the procedures; AMR, MLS performed the experiments. All authors contributed to the writing of the final version of the manuscript. All authors read and approved the final manuscript.

\section{Acknowledgments}

The authors are grateful to all personnel from the Malaria \& Emerging Parasitic Diseases Laboratory (ISCIII-Spain) and the Parasitological Department (MRC-Malaysia) for their comments and support to this study.

\section{References}

1. WHO. World malaria report 2021. Geneva: World Health Organization; 2021. Licence: CC BY-NC-SA 3.0 IGO.

2. Ponce C, Kaczorowski F, Perpoint T, Miailhes P, Sigal A, Javouhey E, et al. Diagnostic accuracy of loop-mediated isothermal amplification (LAMP) for screening patients with imported malaria in a non-endemic setting. Parasite. 2017;24:53.

3. Askling HH, Nilsson J, Tegnell A, Janzon R, Ekdahl K. Malaria risk in travelers. Emerg Infect Dis. 2005;11:436.

4. European Centre for Disease Prevention and Control. Stockholm. Hospital-acquired malaria infections in the European Union. 2018. 
5. Pletsch D. Informe sobre una misión efectuada en España en septiembre-noviembre de 1963 destinada a la certificación de la erradicación del paludismo. Rev San Hig Pública. 1965;39:309-67.

6. Velasco E, Gomez-Barroso D, Varela C, Diaz O, Cano R. Non-imported malaria in non-endemic countries: a review of cases in Spain. Malar J. 2017;16.

7. Chiodini PL. Malaria diagnostics: now and the future. Parasitology. 2014;141:1873-9.

8. Port JR, Nguetse C, Adukpo S, Velavan TP. A reliable and rapid method for molecular detection of malarial parasites using microwave irradiation and loop mediated isothermal amplification. Malar $\mathrm{J}$. 2014;13:454.

9. Patel JC, Lucchi NW, Srivastava P, Lin JT, Sug-aram R, Aruncharus S, et al. Field Evaluation of a RealTime Fluorescence Loop-Mediated Isothermal Amplification Assay, RealAmp, for the Diagnosis of Malaria in Thailand and India. J Infect Dis. 2014;210:1180-7.

10. Cuadros J, Martin Ramírez A, González IJ, Ding XC, Perez Tanoira R, Rojo-Marcos G, et al. LAMP kit for diagnosis of non-falciparum malaria in Plasmodium ovale infected patients. Malar J. 2017;16.

11. Notomi T, Okayami H, Masubuchi H, Yonekawa T, Watanabe K, Amino N, et al. Loop-mediated isothermal amplification of DNA. Nucleic Acids Res. 2000;28.

12. Morris U, Aydin-Schmidt B. Performance and Application of Commercially Available Loop-Mediated Isothermal Amplification (LAMP) Kits in Malaria Endemic and Non-Endemic Settings. Diagnostics. 2021;11:336.

13. Lucchi NW, Gaye M, Diallo MA, Goldman IF, Ljolje D, Deme AB, et al. Evaluation of the Illumigene Malaria LAMP: A Robust Molecular Diagnostic Tool for Malaria Parasites. Sci Rep. 2016;6.

14. Rypien C, Chow B, Chan WW, Church DL, Pillai DR. Detection of Plasmodium Infection by the illumigene Malaria Assay Compared to Reference Microscopy and Real-Time PCR. J Clin Microbiol. 2017;55:3037-45.

15. De Koninck A-S, Cnops L, Hofmans M, Jacobs J, Van den Bossche D, Philippé J. Diagnostic performance of the loop-mediated isothermal amplification (LAMP) based illumigene ${ }^{\circledR}$ malaria assay in a non-endemic region. Malar J. 2017;16.

16. Ljolje $D$, Abdallah R, Lucchi NW. Detection of malaria parasites in samples from returning US travelers using the Alethia ${ }^{\circledR}$ Malaria Plus LAMP assay. BMC Res Notes. 2021;14:128.

17. Frickmann H, Hinz R, Rojak S, Bonow I, Ruben S, Wegner $C$, et al. Evaluation of automated loopmediated amplification (LAMP) for routine malaria detection in blood samples of German travelers A cross-sectional study. Travel Med Infect Dis. 2018;24:25-30.

18. Rubio JM, Post RJ, van Leeuwen WD, Henry M-C, Lindergard G, Hommel M. Alternative polymerase chain reaction method to identify Plasmodium species in human blood samples: the semi-nested multiplex malaria PCR (SnM-PCR). Trans R Soc Trop Med Hyg. 2002;96:199-204.

19. Miguel-Oteo M, Jiram Al, Ta-Tang TH, Lanza M, Hisam S, Rubio JM. Nested multiplex PCR for identification and detection of human Plasmodium species including Plasmodium knowlesi. Asian Pac J Trop Med. 2017;10:299-304. 
20. Santiago Perez MI, Hervada Vidal X, Naveira Barbeito G, Silva LC, Fariñas H, Vázquez E, et al. The Epidat program. Rev Panam Salud Publica. 2010;27:80-2.

21. Barazorda KA, Salas CJ, Bishop DK, Lucchi N, Valdivia HO. Comparison of real time and malachitegreen based loop-mediated isothermal amplification assays for the detection of Plasmodium vivax and P. falciparum. PLoS ONE. 2020;15:e0234263.

22. Meridiam Illumigene Marketing Collateral. Illumigene ${ }^{\circledR}$ Malaria Package Insert, SN11102. REV. 01/16. 2017. Accessed 26 Jan 2022.

23. Meridian Bioscience. Alethia ${ }^{\circledR}$ Malaria and Malaria Plus Package Insert.

24. Lau Y-L, Lai M-Y, Fong M-Y, Jelip J, Mahmud R. Loop-Mediated Isothermal Amplification Assay for Identification of Five Human Plasmodium Species in Malaysia. Am J Trop Med Hyg. 2016;94:336-9.

25. Morris U, Khamis M, Aydin-Schmidt B, Abass AK, Msellem MI, Nassor MH, et al. Field deployment of loop-mediated isothermal amplification for centralized mass-screening of asymptomatic malaria in Zanzibar: a pre-elimination setting. Malar J. 2015;14:205.

26. Singh B, Bobogare A, Cox-Singh J, Snounou G, Abdullah MS, Rahman HA. A genus-and speciesspecific nested polymerase chain reaction malaria detection assay for epidemiologic studies. Am J Trop Med Hyg. 1999;60:687-92. 